\title{
Measuring Situational Awareness at the Small Unit Tactical Level
}

\author{
Gregory Bew, Alexandra Baker, Daniel Goodman, Olivia Nardone, and Michael Robinson \\ United States Military Academy, gregory.bew, alexandra.baker, daniel.goodman, olivia.nardone, \\ michael.robinson@usma.edu
}

\begin{abstract}
The purpose of this study is to propose and validate a method to measure the value of situational awareness at the small-unit tactical level. Currently, the Situational Awareness Global Assessment Technique (SAGAT) is widely considered the best method for measuring situational awareness. However, research on situational awareness is largely focused at the command post level and above, and implementations of SAGAT do not address operations at the small-unit level. In today's Army, technology is a more critical component of modern warfare used to increase the information available to decision makers at all levels. It is presumed that increased levels of available information will facilitate more efficient and effective decisions. However, there are corresponding concerns that such increases will potentially overwhelm the decision maker. We conducted a controlled experiment using the Nett Warrior system and Virtual Battlefield Simulation, Version 2 (VBS2), incorporating a modified form of SAGAT, the Tactical Situational Awareness Test (TSAT), and the principles of cognitive engineering and human factors studies to measure a user's situational awareness. This study finds that TSAT can measure situational awareness at the small-unit tactical level and validates that Nett Warrior increases situational awareness of soldiers in tactical operations.
\end{abstract}

Index Terms - Nett Warrior, SAGAT, TSAT, simulation integration, situational awareness

\section{INTRODUCTION}

Technology is an integral component of modern warfare that is leveraged to provide increasing amounts of information to decision makers. As technology becomes more embedded in all processes, its prevalence continues to filter down to the lowest levels. As more information becomes available to decision makers at all levels, it is becoming increasingly difficult to sort and sift through massive amounts of data. An important question to consider is: will the use of information technologies by tactical units follow a similar path and become more of a burden than an enabler?

The idea of useful information translates directly into the idea of situational awareness. Does information add to a soldier's understanding of their current situation and all its elements? While easy to understand, this concept has proven difficult to measure. The value of information and how to measure that value are highly researched topics.
Much of the work to date has focused on measuring the value of information through simulation. However, the work is largely limited to the evaluation of command and control systems at the battalion and above level. Accordingly, they offer little in the way of measuring the value of a system designed to enhance situational awareness at the dismounted tactical level? What are the measures of performance and effectiveness?

In the beginning stages of the Global War On Terror, many units did not have any devices to help track location or transmit information. As the war progressed, vehicle mounted command and control systems became standard across the force to enable small units. The next step is to enable individual soldiers and dismounted units, by providing them with new technologies.

Extraneous systems on vehicles may or may not be leveraged as intended and the extra burden of size and weight is largely unnoticed. Soldiers do not have that luxury. On a person, any additional piece of equipment is more weight that must be carried, often in place of something else. Therefore, it must be useful; otherwise it likely will not be adopted. This is a key difference between mounted and dismounted systems. "More" is not inherently "better", better is better. "More" in a dismounted network adds to weight and cognitive load. As a result there must be less redundancy and the utility of the equipment must have a more direct impact on soldier effectiveness. How do we measure the value of technology designed to increase situational awareness to ensure we are targeting "better" and not merely adding more?

The efforts to enable dismounted soldiers and help them know more on the battlefield are necessary, but an accepted method to measure benefits of situational awareness is lacking in our test and evaluation process. Systems like Nett Warrior, a small unit command and control system worn by soldiers are in use in the Army today, but they are evaluated in testing by metrics like message completion rate. While that is useful to determine the effectiveness of the network, it does not help in understanding the value of the information or measure any gain or loss of situational awareness. While it is hard to quantify situational awareness, we must develop an accepted way to measure the value of information, how it is presented to a user, and evaluate if it is worth the cost on a soldier's cognitive and physical load.

In this research a method is suggested to measure situational awareness at the small unit level and create a controlled experiment, leveraging simulation and a small 
unit command and control technology. This allows for the measurement of situational awareness during a scenario with and without enabling technologies designed to present a real-time picture of the battle space, to see if the resulting scores from the trials indicated an increase in situational awareness.

After conducting the controlled experiment, the null hypotheses that the situational awareness score for each test subject had the same average, regardless of the inclusion of enabling technology was rejected.

\section{BACKGROUND}

\section{Situational Awareness}

Situational awareness is "the perception of the elements in the environment within a volume of time and space, the comprehension of their meaning and the projection of their status in the near future" [1]. It provides the primary basis for subsequent decision making in dynamic systems.

Increasing situational awareness increases the probability of victory and mission accomplishment. It improves assessments of and responses to combat events. For example, situational awareness can shorten response times and lead to better decision making. Military situations are dynamic, rapid adaption to change is necessary. Situational awareness helps the soldiers adapt to changes on the battlefield.

\section{A Tactical Focus}

This research focuses on the small unit, tactical level. This level is vital because the additional burden of systems that cannot be proven to add value adds to the already high physical and cognitive load on soldiers in combat. Technology included in a soldier's arsenal at the tactical level must provide added value to their operational effectiveness. If this requirement is not met, the added burden to a soldier's load is not mitigated by a significant increase in capabilities.

The United States Army is currently fielding Nett Warrior in order to give soldiers at the small unit level greater situational awareness. To definitively determine the system's utility, measurement techniques must expand beyond the scope of network strength, instead focusing on system utility.

In theory, increasing the information available to a soldier at any given time should enable increases in awareness. It is important to keep in mind, however, that increasing information to the soldier usually necessitates additional equipment, which translates to increased physical and mental burdens. In this experiment, the additional information comes from Nett Warrior system. Specifically, participants will use the Nett Warrior end user device, a Samsung Galaxy Note 2.

Additionally, there is the question of whether or not receiving more information would serve as a distraction or could possibly overwhelm the soldier. In order to make adequate use of the device, the soldier must be able to identify if the incoming information is valuable to their mission quickly.

Battlefield environments also vary greatly depending on the location of the soldier and their role in the mission. Within a tactical operations center (TOC), there is virtually infinite availability to increase information flow. In a TOC, there is enough area space to install more technologies, and these devices do not cause any physical burden to soldiers because they do not need to be transported by an individual. Also, a TOC is not directly executing the mission in real time, and thus can receive more information, filter it, and relay the important information, with less direct impact on mission success.

Soldiers executing missions at the small unit level have a finite amount of weight and equipment they can carry, while still being mission effective. If they were to try to carry more technology to increase their information inflow, it could greatly impact their physical ability to conduct their mission.

\section{Literature Review}

Situational awareness, by nature, is a matter of perception and like most matters of perception, there is a degree of subjectivity related to it. For this reason, measuring situational awareness and how to assess it has been a subject of debate. Leading that debate is the Situational Awareness Global Technique (SAGAT). SAGAT is a freeze probe technique based in goal-directed task analysis, and splits situational awareness into three levels: data (level I), comprehension (level II), and application to the future (level III). Freeze probing means that the test is stopped (frozen) while a brief series of questions are asked and answered quickly. After the questions are answered the test continues. The types of questions asked are derived from the taskanalysis and should ensure to hit all three levels of situational awareness [1].

There are two main concerns generally associated with SAGAT. The first regards freezing the test and its impacts on the test's realism. Richard Pew refutes this critique in "The State of Situational Awareness Measurement", stating the impacts of freezing are negligible since it does not impact the results of the test [2]. However, it is important to keep the freezing unpredictable to the subjects, otherwise they are able to prepare and/or improve their situational awareness [3].

The second concern regards limitations in knowledge based on the times individuals are exposed to information. Mica Endsley notes that "a person's knowledge of the environment...is highly temporal in nature" [1]. Essentially, subject's who are exposed to information for a longer period of time will have more knowledge of that information. If the subject has minimal exposure, they may be unable to recall said information based on its temporal nature. Endsley signals a counter balance to the effect of time, explaining the importance of a subject's confidence level. Essentially, a person's confidence regarding a particular piece of information will greatly impact their ability to recall such 
information [1]. If subjects are presented with easilyunderstood information, the time factor can be counteracted by their high confidence level regarding the particular data.

\section{METHODOLOGY}

\section{Measuring Situational Awareness}

SAGAT, as is, is not entirely applicable for any and all scenarios, to include $\mathrm{C} 4 \mathrm{i}$ (command, communications, computers, and intelligence) scenarios. Since most new situational awareness technologies, like Nett Warrior, fall under the $\mathrm{C} 4 \mathrm{i}$ category, and since no technique for specifically analyzing situational awareness for $\mathrm{C} 4 \mathrm{i}$ currently exists, a new method of analyzing C4i must be developed.

To measure situational awareness of $\mathrm{C} 4 \mathrm{i}$ systems, we adapted the SAGAT approach to create the Tactical Situational Awareness Test (TSAT). TSAT assessed situational awareness using probes based on random control measures, called "phase lines." At these random phase lines, subjects are asked a series of questions based on their understanding of the scenario they are executing, with their answers being compared to the "ground truth"(the correct answer) to assess if they understand the situation. Each question receives a binary score, either 1 for correct or 0 for incorrect. The sum of all individual question scores make up the subject's situational awareness score as in (1).

$$
\text { SA Score }=\sum_{i=1}^{n}\left(x_{i}\right)
$$

\section{A Controlled Experiment}

A controlled experiment was selected in order to evaluate if the TSAT method could measure situational awareness. For this experiment, it was determined that a virtual simulation was the best approach before conducting a more resourceintensive field experiment. A controlled experiment using simulation gave an effective, inexpensive, and malleable method to attempt to measure situational awareness. The virtual simulation of the controlled experiment provided adequate initial results for a fraction of the cost and over a far shorter time period. Upon deciding to use a virtual simulation, a simulation platform and situation awareness focused technology needed to be identified.

The research team integrated a program called Virtual Battlefield Simulator, version two (VBS2) and Nett Warrior to create the described test environments for the controlled experiment. We constructed two scenarios using the Shelby and McKenna urban warfare training sites located at Fort Benning, GA. These training areas were deliberately chosen since they are validated training environments used by the Army. Two maps were utilized, one for training and one for the test scenario. Eleven groups of three cadets conducted a single training mission, then two test missions for a total of 22 trials tested.

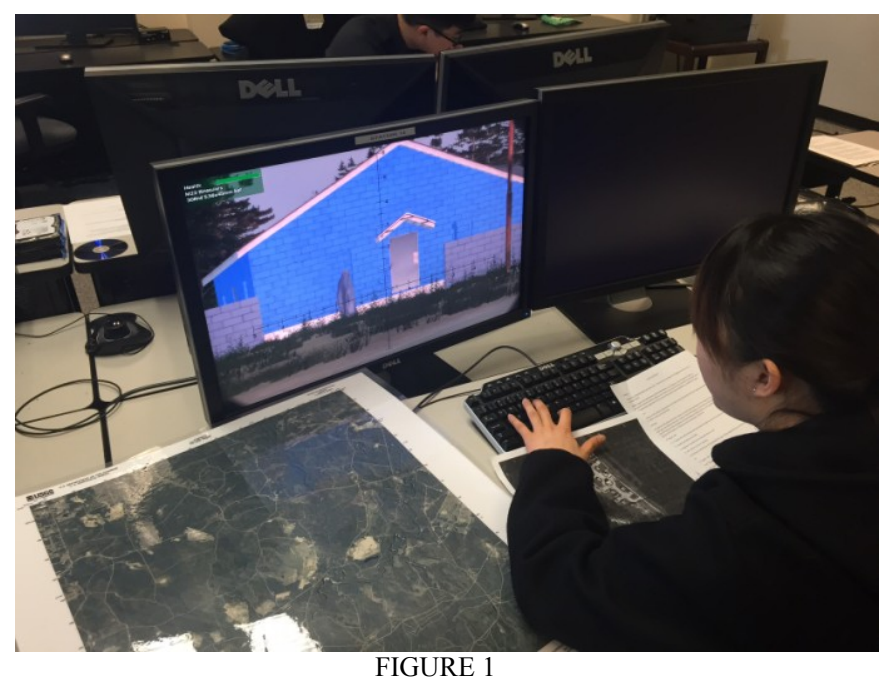

CADET EXECUTING CONTROLLED EXPERIMENT

III. Data Protocol Translation

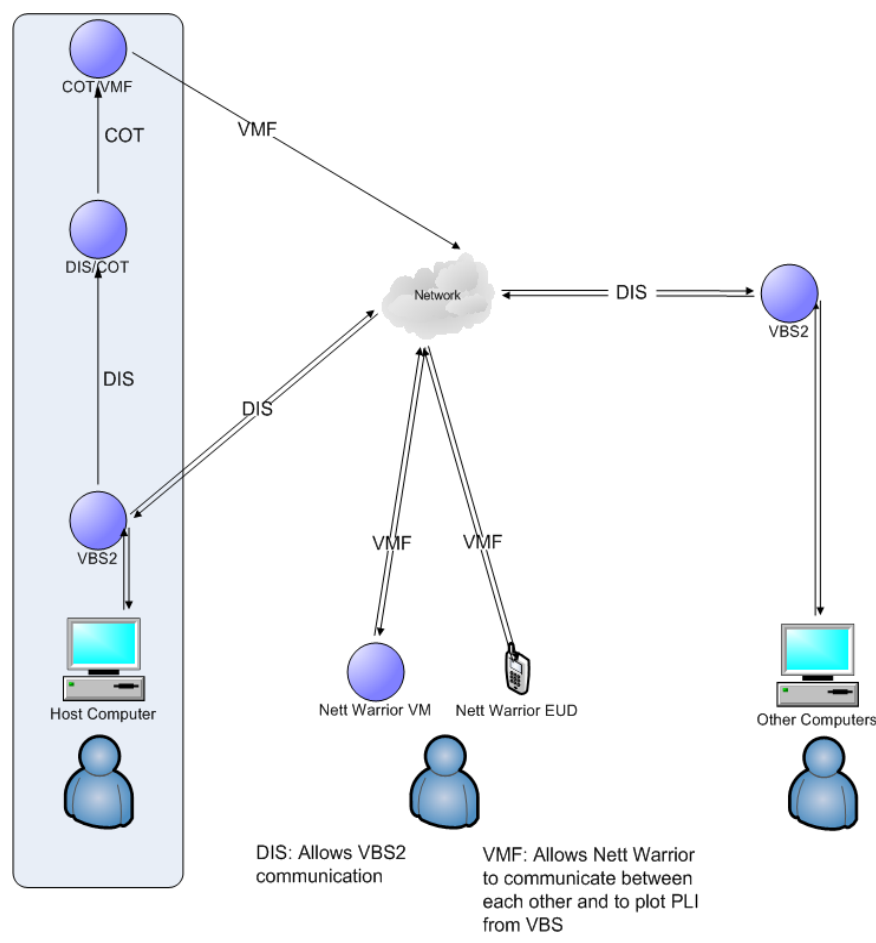

FIGURE 2

DATA Flow DiAgRAM BETWEEN VBS2 TO NETT WARRIOR

To effectively conduct a controlled experiment with Nett Warrior, multiple software programs needed to communicate. Essentially, data packets need to flow between the host computer, alternate computers, VBS2, Nett Warrior, and the central network. These data packets also needed to be translated into the correct protocols for use by each system. This software integration allowed the user to physically interact with Nett Warrior while participating in controlled, virtual experiments in VBS2. 
The host computer sends out the initial data packets using the DIS (distributed interactive simulation) protocol. This initial data originates in the host computer on VBS2, where the scenario specifications, player classifications, and other basic parameters exist. Data packets originating from the host computer using DIS protocol moved to the wireless network. This network linked together the host computer, Nett Warrior, Nett Warrior VM (Virtual Machine), along with the other computer used in the experiments. These original DIS packets are used to communicate between VBS2 so the player can conduct the multiplayer experiment. At the same time, the DIS packets are translated internally in the host computer to the CoT protocol (Cursor on Target), and again to the VMF (Variable Message Format) protocol. The internal translator used a URN table (Unit Role Number) to specify which entities in the VBS2 simulation needed to be translated to the VMF protocol for representation on Nett Warrior. This URN table matched the many DIS UIDs (Unit Identifications) with the requested Nett Warrior URNs.

Simultaneously, Nett Warrior and the Nett Warrior virtual machine (if used) send VMF protocol data packets directly to the network. The Nett Warrior VMF data packets never directly interact with VBS2, but rather interact with data packets originating from VBS2 DIS that are translated to VMF. VBS2 traffic to VMF is outbound only.

To improve the effectiveness of the controlled experiment, improvements are currently being explored. Specifically, an ideal situation would constitute data packets moving directly from the host computer to the network using the CoT protocol. This will provide added benefit to include continual location display on Nett Warrior and more accurate player identification icons to allow the user to better visualize their location in the VBS2 scenario. However, this current setup met the needs for the controlled experiment. The described setup is displayed in Figure 2.

\section{Probe Development}

In order to measure the situational awareness of test subjects, a series of probes were developed and deployed at various points in the experiment. The series of probes provided a standardized questionnaire presented to every participant. Five uniform sets of questions were asked at five points throughout the scenario: prior to initiation of movement, at three predetermined phase lines during the scenario, and following mission completion. These phase lines were placed in the mission, without the knowledge of the participant so they did not know when a pause might happen.

When determining the most effective probes to present to the subjects, multiple factors were considered. These factors included physical condition, mission comprehension, identifying surrounding personnel and units, and location data. The questions directly targeted these factors. In addition, the probes asked questions that reflected all three levels of situational awareness. This allows us to identify not only memorization of information, but also understanding and projection.

The measurement technique used in this experiment hinges on the effectiveness of the developed probes and the comparison of probe responses to the actual state of the subjects at each phase line.

\section{Scenario Development}

Many factors were considered to ensure that the scenario used in experimentation was realistic in nature. A realistic scenario requires test subjects to think in a tactical manner and make well-reasoned decisions.

Each group of cadets conducted a familiarization trial using the McKenna scenario to help them get familiar with both Nett Warrior and VBS2. The cadets' situational awareness was measured during two trials conducted on the Shelby scenario. One cadet was designated as the Squad Leader, and the other two cadets as Team Leaders. We ran the two Shelby trials consecutively, and randomized whether or not the group had Nett Warrior at their disposal for either the first or the second trial. This randomization was to help identify the effects of experiential learning, if any existed, that might occur during the trials.

Additionally, all test subjects were provided with an operations order detailing the initial mission, an area map, and a gridded reference graphic (GRG). Subjects had these items at their disposal through every trial to allow a baseline of situational awareness.

This area of operation used in the controlled experiment, simulated through the Shelby map, provided an excellent avenue for the experiment. The participant in each trial is part of a squad that is tasked to move from the southern portion to the northern portion of the town, engaging with the population and collecting basic information and intelligence. The town consists of buildings, vehicles, roads, non-hostile, and hostile personnel. One enemy fighter was placed behind a building toward the southern end of the map, forcing the squad to modify its original plan of action to engage this target during movement Additionally, a civilian in the middle of the village was simulated to provide intelligence to the squad concerning a hostage being held at the northern-most building in the town. The task of the friendly squad then shifts to a hostage recovery mission, forcing the subjects to maneuver to the building, engage enemy forces, and recover the hostage. The purpose for the change was to add realism in the form of battlefield dynamics and confusion. To test a subject's situational awareness, the scenario must be dynamic. This ensures that a subject's understanding is not due to memorization of the original mission guidance.

At three pre-determined phase lines from south to north in the village, the scenario was paused to allow the proctor to present the probes for questioning. This allowed the analysis of the subject's situational awareness through the controlled experiment, as conditions changed.

Finally, when each trial was complete, each cadet completed an individual NASA Task Load Index (TLX) [4], 
which provided randomized questions regarding selfperceived mental demand, physical demand, temporal demand, effort, performance, and frustration level they experienced during the trial. The TLX then delivered a results matrix of data that was used to compare how cadets self-reported their feelings between the trials with or without the Nett Warrior device.

\section{RESULTS}

The score of the individuals in each trial was totaled and used for analysis. The data analysis showed statistically significant results that there is a difference in the mean of scores for a subject with and without Nett Warrior.

First the scores were divided into two paired groups. The first group was the participant's first trial, the second group was the participant's second trial. This created four different groups: first trial enabled, first trial traditional, second trial enabled, second trial traditional. Using analysis of variance (ANOVA) these groups were compared to see if there was a statistical difference between the scores of participants who were enabled on their first trial and those that were enabled on their second trial, and similarly those that were traditional on their first trial, and those traditional on their second trial. Then, blocking by trial number, the results yielded that there were no significant effects of experiential learning in the results, allowing for a paired ttest to be conducted on just two main groups: the situational awareness scores of trials with Nett Warrior compared to those from traditional trails.

A paired t-test was used to analyze the mean average difference between two samples as in (2). For this experiment, the test was conducted using the null hypothesis that the average situational awareness score between the two groups is the same. The mathematical representation of the hypothesis is shown below in (3) and (4), where $\mu_{D}$ is the average mean difference between the two data sets.

$$
\begin{aligned}
& t_{0}=\frac{\overline{\mathrm{d}}-\Delta_{0}}{s_{\mathrm{d}} / \sqrt{\mathrm{n}}} \\
& H_{0}: \mu_{D}=0 \\
& H_{a}: \mu_{D} \neq 0
\end{aligned}
$$

The results of this test, shown in Figure 3, showed that at the $95 \%$ confidence level, the p-value of our analysis was 2.196e-09. The p-value of a statistical test represents the probability of inaccurately rejecting the null hypothesis when it is actually true. This extremely small p-value supports our conclusion to reject the null hypothesis that the average of the differences between the two samples is equal to zero.

A visual representation of our results, shown in Figures 3 , 4, and 5, show a higher median value for trials with Nett Warrior. Additionally, the lower quartile of the data with Nett Warrior is on line with the upper bound of the data without the device. This shows the majority of scores of teams using Nett Warrior were higher than the highest score achieved by a team without Nett Warrior.

\begin{tabular}{|c|}
\hline Paired T Test \\
\hline $\mathrm{t}=9.4506$ \\
\hline $\mathrm{p}$-value $=2.196 \mathrm{e}-09$ \\
\hline \\
\hline 95\% Confidence Interva $\mathrm{:}$ \\
\hline$(3.580076,5.586590)$ \\
\hline Mean of the Differences \\
\hline 4.583333 \\
FIGURE 3 \\
PAIRED T RESULTS
\end{tabular}
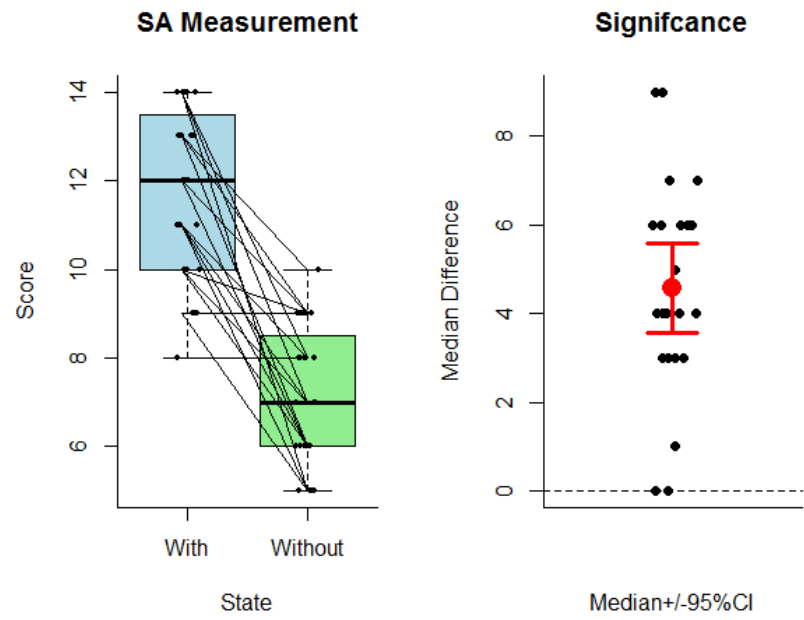

FIGURE 4

BOX PLOT OF TRIAL SCORES

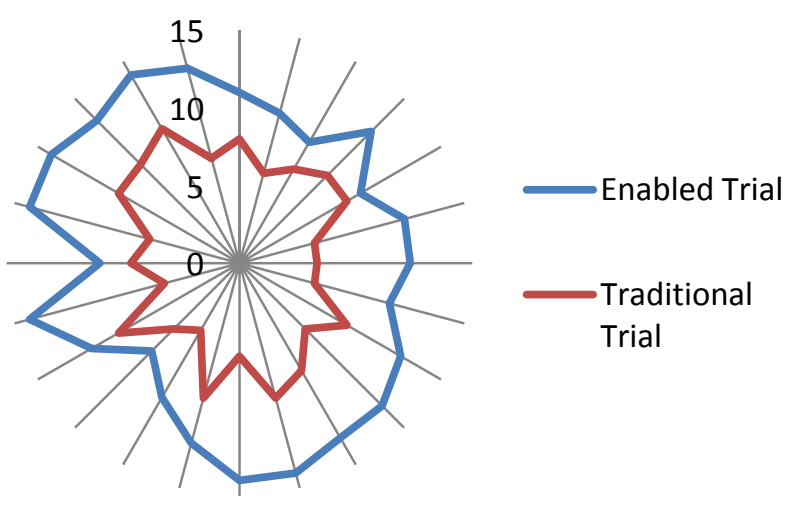

FIGURE 5

RADAR PLOT OF TRIAL SCORES

\section{CONCLUSIONS AND RECOMMENDATIONS}

This research has led to several distinct conclusions. First, the results rejected the null hypothesis that there is no significant difference in situational awareness scores in traditional and technology enables users. This strongly supports that TSAT is a viable method to measure situational awareness and that it warrants further research.

The manipulation of the SAGAT method proved effective in measuring situational awareness at the small 
unit, tactical level. This provides justification for small units throughout the operational force of the US Military to expand and employ similar methods to assess situational awareness in training environments.

Also, an important conclusion from this study is that our results support the statement that situational awareness can be measured, and a statistically significant difference is detected when the user has more information.

This research provides an excellent base of data that can be expanded upon through further controlled trials and follow-on experimentation in a field test.

\section{FUTURE WORK}

There are many potential implications of this research. Future work can be conducted in several areas.

First, this method will be tested in a field experiment at the United States Military Academy. Two cadet platoons, a control platoon and an experimental platoon, will conduct a twelve day cycle of missions. The control platoon will be issued traditional infantry platoon equipment, while the experimental platoon is issued the Nett Warrior system. Each platoon will answer probes at various points throughout the training to assess their situational awareness.

Second, a larger controlled experiment should be conducted to create a baseline situational awareness score for traditional units. With one standard baseline score, other equipment can be tested against a baseline with no need to replicate a "without" phase every experiment. This would allow different technologies to be assessed against the baseline, as long as the same scenario and probes are used each time with different participants.

Third, an experiment that determines the value of the situational awareness provided by Nett Warrior and other tools specifically. In this experiment, participants used Nett Warrior as their command and control enabling technology, but they did not receive formal training on the device. Therefore, this experiment did not leverage all of the capabilities of the system and only served as a proof of concept. Now a more in depth study can be done to create "scores" for different alternatives to measure how much they aid in developing situational awareness.

Fourth, in follow on trials, extra emphasis needs to be placed on probe development. The test is only as good as the questions that are asked, so more work needs to be done to tailor questions to evaluate specific technologies, mission, and environments. Ultimately, a standardize battery of probes would be ideal, but may not be possible due to the dynamic nature of the military and diverse mission focuses.

\section{ACKNOWLEDGMENT}

The authors would like to acknowledge the Nett Warrior office for supporting this research with time and equipment that were vital to conducting this study. Brian Brennan, Erin Connors, Mark Frye, and Steve Henderson, thank you so much for your hours of support.

\section{REFERENCES}

[1] Endsley, Mica. "Toward a Theory of Situation Awareness in Dynamic Systems." Human Factors 37 (1.)1995.

[2] Pew, Richard. "The State of Situational Awareness Measurement: Heading Toward the Next Century." Situational Awareness: Analysis and Measurement. Mahwah, NJ: Lawrence and Erlbaum Associates, 2000.

[3] Endsley, Mica. "Situational Awareness Global Assessment Technique (SAGAT)." Aerospace and Electronics Conference 1988. Dayton, $\mathrm{OH}$.

[4] Hart, Sandra and Staveland, Lowell. "Development of NASA-TLX (Task Load Index): Results of Empirical and Theoretical Research." Human Mental Workload 1988.

\section{AUTHOR INFORMATION}

Gregory Bew, Instructor, Department of Systems Engineering, United States Military Academy.

Alexandra Baker, Cadet, United States Military Academy.

Daniel Goodman, Cadet, United States Military Academy.

Olivia Nardone, Cadet, United States Military Academy.

Michael Robinson, Cadet, United States Military Academy. 